\title{
Stabilisation of Spectral/hp Element Methods Through Spectral Vanishing Viscosity: Application to Fluid Mechanics Modelling
}

\author{
Robert M. Kirby ${ }^{\mathrm{a}}$, Spencer J. Sherwin ${ }^{\mathrm{b}, 1}$

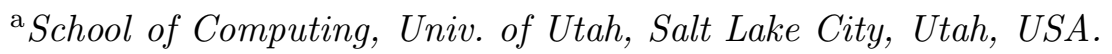 \\ ${ }^{\mathrm{b}}$ Department of Aeronautics, Imperial College London, London, UK.
}

\begin{abstract}
In this paper we present a formulation of spectral vanishing viscosity (SVV) for the stabilisation of spectral $/ h p$ element methods applied to the solution of the incompressible Navier-Stokes equations. We construct the SVV around a filter with respect to an orthogonal expansions, and prove that this methodology provides a symmetric semi-positive definite SVV operator. After providing a few simple oneand two-dimensional examples to demonstrate the utility of the SVV, we examine how it can be applied to a spectral/ $h p$ element discretisation of the Navier-Stokes equations using a velocity correction splitting scheme. We provide three fluid flow examples to help illustrate the pros and cons of this approach on stability and accuracy.
\end{abstract}

Keywords: Spectral/hp Elements, Polynomial De-Aliasing, Spectral Vanishing Viscosity, Stabilisation

\section{Introduction}

The computational fluid mechanics literature is replete with examples of the addition of mechanisms to stabilise solution techniques for the Navier-Stokes equations. The simplest of these mechanisms is the addition of second-order dissipative operators as advocated by von Neumann and Richtmyer [1]. The addition of such dissipative operators in the context of high-order numerical methods is of great interest. In particular, the formulation of

Email addresses: kirby@cs.utah.edu (Robert M. Kirby), s.sherwin@imperial.ac.uk (Spencer J. Sherwin).

1 Corresponding Author

Preprint submitted to Comp Meth Appl Mech EngrgSubmitted May 30, 2004/Revised September 15, 2004 
dissipative operators which provide sufficient dissipation to stabilise the solution while not degrading significantly the convergence properties of the method are desired. As an alternative to the introduction of additional dissipative (stabilising) operators to the original governing equations, Kirby et al. [2] showed that both stability and accuracy of spectral/hp element discretisation of the incompressible Navier-Stokes equations can be greatly influenced through consistent-integration of the nonlinear terms [3]. Exact integration provides a true Galerkin projection of the nonlinear terms and hence removes aliasing errors (i.e. it is essentially polynomial de-aliasing). However one concern of applying consistent-integration is that it is costly. In the case of the incompressible Navier-Stokes, $O\left([3 P / 2]^{\text {Dim }}\right)$ instead of $O\left([P]^{\text {Dim }}\right)$ points per element (where $P$ is the polynomial order) are necessary to accurately integrate the nonlinear terms. This notably increases the total number of floating point operations (and storage) required. A further concern is that aliasing due to the nonlinear terms may not be the only source of instability within the simulation.

In light of these observations, many have questioned whether there are alternative means of stabilising the solution which are both inexpensive and do not degrade accuracy. It has been proposed that polynomial filtering [4] may provide a more computationally efficient means of removing the effects of polynomial aliasing and other destabilising influences. Such filtering techniques have been successfully employed to stabilise spectral element polynomial methods [5-7].

The purpose of this paper is to formulate a symmetric, positive semi-definite spectral vanishing viscosity (SVV) method for spectral/hp element methods in hybrid shaped domains extending the work of [8]. In detailing this formulation we demonstrate the requirements on the filter projection to maintain discrete symmetry of the SVV operator and provide fluid flow examples which illustrate the pros and cons of this methodology.

\subsection{Outline}

The layout of this paper is as follows. In Section 2 we provide the mathematical and algorithmic description of the spectral vanishing viscosity method. We first provide the motivation for the methods from Fourier methods and then proceed to outline spectral vanishing viscosity (SVV) for spectral/hp element methods. We then provide two demonstrative examples in one-dimension and in two-dimensions which help elucidate the concepts. In Section 3 we outline how the SVV can be added to a velocity correction splitting scheme to discretise the incompressible Navier-Stokes equations. In Section 4 we provide three demonstrative fluid flow examples demonstrating the effects of SVV. We conclude in Section 5 with a summary of this work and a discussion of future challenges. 


\section{Mathematical and Algorithmic Description}

\subsection{Motivation}

Tadmor [9] first introduced the concept of spectral vanishing viscosity (SVV) using the inviscid Burgers equation

$$
\frac{\partial}{\partial t} u(x, t)+\frac{\partial}{\partial x}\left[\frac{u^{2}(x, t)}{2}\right]=0,
$$

subject to given initial and boundary conditions. The distinct feature of solutions to this problem is that spontaneous jump discontinuities (shock waves) may develop, and hence $a$ class of weak solutions can be admitted. Within this class, there are many possible solutions, and in order to single out the physically relevant solution an additional entropy condition is applied, of the form

$$
\frac{\partial}{\partial t}\left[\frac{u^{2}(x, t)}{2}\right]+\frac{\partial}{\partial x}\left[\frac{u^{3}(x, t)}{3}\right] \leq 0 .
$$

In numerical implementations, spectral methods are often augmented with smoothing procedures in order to reduce the Gibbs oscillations [10] associated with discontinuities arising at the domain boundaries or due to under-resolution. However, with nonlinear problems, convergence of the Fourier method, for example, may fail despite additional smoothing of the solution. Tadmor [9] introduced the spectral vanishing viscosity method, which adds a small amount of controlled dissipation that satisfies the entropy condition, yet retains spectral accuracy. It is based on viscosity solutions of nonlinear Hamilton-Jacobi equations, which have been studied systematically in [11]. Specifically, the viscosity solution for the Burgers equation has the form

$$
\frac{\partial}{\partial t} u(x, t)+\frac{\partial}{\partial x}\left[\frac{u^{2}(x, t)}{2}\right]=\epsilon \frac{\partial}{\partial x}\left[Q_{\epsilon} \frac{\partial u}{\partial x}\right],
$$

where $\epsilon(\rightarrow 0)$ is a viscosity amplitude and $Q_{\epsilon}$ is a viscosity kernel, which may be nonlinear and, in general, a function of $x$. Convergence may then be established by compensated compactness estimates combined with entropy dissipation arguments [9]. To respect spectral accuracy, the SVV method makes use of viscous regularisation and equation (3) may be rewritten in discrete form (retaining $N$ modes) as

$$
\frac{\partial}{\partial t} u_{N}(x, t)+\frac{\partial}{\partial x}\left[\mathcal{P}_{N}\left(\frac{u^{2}(x, t)}{2}\right)\right]=\epsilon \frac{\partial}{\partial x}\left[Q_{N} * \frac{\partial u_{N}}{\partial x}\right]
$$

where the $\operatorname{star}(*)$ denotes convolution and $\mathcal{P}_{N}$ is a projection operator. $Q_{N}$ is a (possibly nonlinear) viscosity kernel, which is only activated for high wave numbers. In Fourier space, this kind of spectral viscosity can be efficiently implemented as multiplication of the Fourier coefficients of $\hat{u}_{N}$ with the Fourier coefficients of the kernel $\hat{Q}_{N}$, i.e.,

$$
\epsilon \frac{\partial}{\partial x}\left[Q_{N} * \frac{\partial u_{N}}{\partial x}\right]=-\epsilon \sum_{P_{\text {cut }} \leq|k| \leq N} k^{2} \hat{Q}_{k}(t) \hat{u}_{k}(t) e^{i k x}
$$


where $k$ is the wave number, $N$ the number of Fourier modes, and $P_{\text {cut }}$ the wavenumber above which the spectral vanishing viscosity is activated.

Originally, Tadmor [9] used

$$
\hat{Q}_{k}=\left\{\begin{array}{l}
0,|k| \leq P_{\text {cut }} \\
1,|k|>P_{\text {cut }},
\end{array}\right.
$$

with $\epsilon P_{\text {cut }} \sim 0.25$ based on the consideration of minimising the total-variation of the numerical solution. In subsequent work, however, a smooth kernel was used, since it was found that the $C^{\infty}$ smoothness of $\hat{Q}_{k}$ improves the resolution of the SVV method. For Legendre pseudo-spectral methods, Maday, Kaber \& Tadmor [12] used $\epsilon \approx N^{-1}$, activated for modes $k>P_{\text {cut }} \approx 5 \sqrt{N}$, with

$$
\hat{Q}_{k}=e^{-\frac{(k-N)^{2}}{\left(k-P_{\text {cut }}\right)^{2}}}, \quad k>P_{\text {cut }} .
$$

Karamanos \& Karniadakis [8] made the first extension of the spectral vanishing viscosity concept to spectral/hp element methods. In [8], the general form of the SVV operation as presented by Tadmor is maintained; however, polynomial filtering is used to mimic the convolution operator in Tadmor's formulation. In this work, SVV filtering was applied directly to the $C^{0}$ hierarchical (linearly independent but non-orthogonal) basis. Kirby \& Karniadakis [13] proposed SVV filtering with respect to orthogonal expansions, and demonstrated the concept in the context of LES modelling on incompressible turbulent channel flows. Xu \& Pasquetti [14] formulated SVV for nodal spectral elements and demonstrated the stabilisation effect within the context of cylinder flows. Finally Sirisup \& Karniadakis [15] have demonstrated the use of SVV stabilisation in the context of principle component analysis.

We present in the next section a formulation of SVV for spectral/hp elements [3] using a continuous Galerkin formulation which filters on an orthogonal basis. This work extends the concepts mentioned in [13] by formulating SVV using orthogonal expansions for one-, twoand three-dimensional spectral element discretisations, and we further demonstrate that the operator obtained is symmetric and semi-positive definite.

\subsection{SVV for Spectral/hp Element Methods}

We define the multi-dimensional SVV operator over the solution domain as

$$
S_{V V}(u)=\epsilon \sum_{i=1}^{\operatorname{Dim}} \frac{\partial}{\partial x_{i}}\left[Q_{\operatorname{Dim}} \star \frac{\partial u}{\partial x_{i}}\right]
$$

To develop the spectrally vanishing viscosity approach in a multi-dimensional polynomial expansion, as typically applied in a spectral/hp element expansion, we need to construct the Galerkin projection of equation (7). Following standard finite element construction we take the inner product of $(7)$ with respect to a $C^{0}$ continuous test function $v$ and apply the 
divergence theorem to arrive at an elemental operator of the form

$$
S_{V V}^{e}(v, u)=\epsilon \sum_{i=1}^{\operatorname{Dim}}\left(\frac{\partial v}{\partial x_{i}}, Q_{\operatorname{Dim}} \star \frac{\partial u}{\partial x_{i}}\right)_{\Omega^{e}}
$$

where $(,)_{\Omega^{e}}$ denotes the Legendre inner product in the elemental region $\boldsymbol{x} \in \Omega^{e}$. In the above expression we understand that the expansion spaces of $v, u$ are both in a globally $C^{0}$ continuous space and so the element operator $S_{V V}^{e}(v, u)$ can be assembled into a global operator using the standard global assembly process, see [3].

To illustrate the construction and numerical implementation of the operator (8), in section 2.2.1 we introduce some matrix notation which can be applied to construct the elemental Laplacian operator, following [3]. Then in section 2.2.2 we use the matrix notation to construct the SVV filter and further demonstrate that a projection to an orthogonal elemental basis is necessary to maintain the symmetry of the SVV operator.

\subsubsection{Matrix formulation of the Laplacian operator}

We consider the elemental weak Laplacian matrix which arises from a standard Galerkin projection of the form

$$
\boldsymbol{L}^{e}[j][i]=\left(\nabla \phi_{j}, \nabla \phi_{i}\right)_{\Omega^{e}} .
$$

In equation $(9), \phi_{i}(\boldsymbol{x}), \boldsymbol{x}=\left[x_{1}, x_{2}, x_{3}\right]$ is a multi-dimensional expansion basis which could be either modal or nodal in form and spans a standard polynomial space within the mapped standard finite element region. In a standard spectral/hp element implementation this inner product in equation (9) is typically evaluated using Gaussian quadrature, the zeros of which we denote by a set of points $\boldsymbol{x}_{j}$. We can therefore introduce a basis matrix $\boldsymbol{B}$ which has columns of $\phi_{i}(\boldsymbol{x})$ evaluated at the quadrature points $\boldsymbol{x}_{j}$, i.e.

$$
\boldsymbol{B}[j][i]=\phi_{i}\left(\boldsymbol{x}_{j}\right)
$$

The location of the points $\boldsymbol{x}_{j}$ is clearly dictated by the type and order of the Gaussian quadrature adopted. We note that if a nodal expansion, based on the Lagrange cardinal function, is chosen through a set of Gauss-Lobatto-Legendre quadrature points (as is typically the case in standard spectral element methods) then $\boldsymbol{B}$ becomes an identity matrix, i.e. $\boldsymbol{B}=\boldsymbol{I}$. Consistent with the definition of $\boldsymbol{B}$ we can define a derivative matrix $\boldsymbol{D}_{l}$ which evaluates the derivative of $\phi_{i}(\boldsymbol{x})$ in the $x_{l}$ direction at the quadrature points, i.e.

$$
\left(\boldsymbol{D}_{l} \boldsymbol{B}\right)[j][i]=\left.\frac{\partial \phi_{i}}{\partial x_{l}}\right|_{\boldsymbol{x}_{j}} \quad \text { or }\left.\quad \frac{\partial \phi_{i}}{\partial x_{l}}\right|_{\boldsymbol{x}_{j}}=\sum_{k} \boldsymbol{D}_{l}[j][k] \boldsymbol{B}[k][i] .
$$

Finally we define a diagonal matrix $\boldsymbol{W}$ whose diagonal contains the quadrature weights multiplied by the Jacobian of the mapping from $\Omega^{e}$ to a standard region $\Omega^{s t}$ evaluated at the quadrature points $\boldsymbol{x}_{j}$. The elemental weak Laplacian matrix (9) can be defined in terms 
of the above matrices as:

$$
\boldsymbol{L}^{e}=\sum_{l=1}^{\operatorname{Dim}} \boldsymbol{B}^{T} \boldsymbol{D}_{l}^{T} \boldsymbol{W} \boldsymbol{D}_{l} \boldsymbol{B}
$$

where Dim is the dimension of the problem under consideration. The evaluation of the Laplacian matrix, denoted by the matrix operations in equation (10), is exact for a polynomial expansion bases providing a sufficiently high quadrature order is adopted and the elemental mapping to the standard region is linear. Even if the mapping is nonlinear, providing it is well behaved the evaluation of the Laplacian from equation (10) will be consistent with the overall spectral/ $h p$ element approximation.

We note that whilst the above matrix notation is convenient to highlight the operations required to evaluate the Laplacian matrices, the operations represented by the matrix multiplications are typically more efficiently evaluated using a tensorial structure of the expansion bases. For example, in spectral/ $h p$ element discretisations the basis $\phi_{i}(\boldsymbol{x})$ is often constructed using tensor product of one-dimensional polynomial expansions. In this case the operations of the matrix $\boldsymbol{B}$ can be efficiently evaluated using the sum factorisation technique [3]. Having evaluated the expansion basis at a tensorial set of quadrature points, it is then possible to use Kronecker delta property of the Lagrange polynomial through the quadrature points to evaluate the derivatives as a series of one-dimensional operations. See [3] for more details on these implementation details.

\section{Alternative Matrix Construction of the Laplacian System}

An alternative matrix construction of the Laplacian system to that shown in equation (10) is

$$
\boldsymbol{L}^{e}=\sum_{l=1}^{\operatorname{Dim}} \boldsymbol{S}_{l}^{T} \boldsymbol{M}^{-1} \boldsymbol{S}_{l}
$$

where

$$
\boldsymbol{M}=\boldsymbol{B}^{T} \boldsymbol{W} \boldsymbol{B} \quad \boldsymbol{S}_{l}=\boldsymbol{B}^{T} \boldsymbol{W} \boldsymbol{D}_{l} \boldsymbol{B} .
$$

As we shall see in section 2.2.2, the construction shown in equation (11) is convenient for the interpretation of the SVV operator. We note that under the assumption of exact numerical integration (since we are only considering polynomial expansions) the elemental mass matrix $\boldsymbol{M}$ and elemental matrix $\boldsymbol{S}$ are equivalent to the analytical operations

$$
\boldsymbol{M}[j][i]=\left(\phi_{j}, \phi_{i}\right)_{\Omega^{e}} \quad \boldsymbol{S}_{l}[j][i]=\left(\phi_{j}, \frac{\partial \phi_{i}}{\partial x_{l}}\right)_{\Omega^{e}} .
$$

To demonstrate the equivalence between the equations (10) and (11) we first note that $\boldsymbol{M}^{-1} \boldsymbol{S}_{l}$ is the Galerkin projection of the derivative of the expansion basis $\partial \phi_{i} / \partial x_{l}$ onto the original expansion basis $\phi_{i}(\boldsymbol{x})$, i.e.

$$
\left(\phi_{i}(\boldsymbol{x}), \sum_{j} \hat{v}_{j} \phi_{j}(\boldsymbol{x})\right)_{\Omega^{e}}=\left(\phi_{i}(\boldsymbol{x}), \sum_{j} \hat{u}_{j} \frac{\partial \phi_{j}}{\partial x_{l}}\right)_{\Omega^{e}} \Rightarrow \hat{\boldsymbol{v}}=\boldsymbol{M}^{-1} \boldsymbol{S}_{l} \hat{\boldsymbol{u}}
$$


where $\hat{\boldsymbol{u}}[i]=\hat{u}_{i}$ and $\hat{\boldsymbol{v}}[i]=\hat{v}_{i}$. When the expansion basis is a polynomial in the elemental region $\Omega^{e}$, which typically implies that the mapping to the standard region is linear, the derivative of the expansion basis will also lie within the space of the original expansion. Therefore under a linear elemental mapping and using exact numerical differentiation and differentiation we also note that

$$
\boldsymbol{D}_{l} \boldsymbol{B}=\boldsymbol{B} \boldsymbol{M}^{-1} \boldsymbol{S}_{l}
$$

In general we interpret equation (13) as a Galerkin projection of the derivative of $\phi_{i}(\boldsymbol{x})$ back onto the expansion basis. Therefore even if the expansion is not a polynomial in $\Omega^{e}$, for example in a curvilinear domain, the matrix operation $\boldsymbol{B} \boldsymbol{M}^{-1} \boldsymbol{S}_{l}$ represents an approximation of the derivative of the expansion basis consistent with the overall spectral/hp discretisation.

Finally inserting equation (13) into equation (10) we obtain

$$
\boldsymbol{L}^{e}=\sum_{l=1}^{\text {Dim }} \boldsymbol{B}^{T} \boldsymbol{D}_{l}^{T} \boldsymbol{W} \boldsymbol{D}_{l} \boldsymbol{B}=\sum_{l=1}^{\text {Dim }} \boldsymbol{S}_{l}^{T} \boldsymbol{M}^{-T} \boldsymbol{B}^{T} \boldsymbol{W} \boldsymbol{B} \boldsymbol{M}^{-1} \boldsymbol{S}_{l}
$$

Since the mass matrix is symmetric, $\boldsymbol{M}^{-T}=\boldsymbol{M}^{-1}$ and using the fact that $\boldsymbol{M}=\boldsymbol{B}^{T} \boldsymbol{W} \boldsymbol{B}$ we obtain

$$
\boldsymbol{L}^{e}=\sum_{l=1}^{\operatorname{Dim}} \boldsymbol{S}_{l}^{T} \boldsymbol{M}^{-1} \boldsymbol{S}_{l}
$$

\subsubsection{Spectral/hp Element Spectrally Vanishing Viscosity}

We now turn our attention to the construction of the spectral/hp SVV operator as defined in equation (8) which as we have previously observed is similar in form to the Laplacian operator with a viscosity kernel $Q_{\text {Dim }}$. As discussed in section 2.1 the viscosity kernel need not be explicitly evaluated in physical space but can be interpreted as a convolution operator in a modal space. We therefore would like to project the derivative of the expansion to a modal space and apply an analogous polynomial SVV convolution operator.

We have already observed that $\boldsymbol{M}^{-1} \boldsymbol{S}_{l}$ in equation (12) is equivalent to projecting the derivative of the expansion basis in the $x_{l}$ direction on to the same expansion basis. We can generalise this projection on to any basis which spans the same polynomial space. Denoting by $\tilde{\phi}_{i}(\boldsymbol{x}), 0 \leq i<N_{\text {mod }}$ a polynomial expansion which spans the same space as $\phi_{i}(\boldsymbol{x})$, $0 \leq i<N_{\text {mod }}$ we can define a basis matrix $\tilde{\boldsymbol{B}}$ in an analogous fashion to $\boldsymbol{B}$, i.e.

$$
\tilde{\boldsymbol{B}}[j][i]=\tilde{\phi}_{i}\left(\boldsymbol{x}_{j}\right) .
$$

Following the construction leading to equation (12) we note that the projection of the derivative of $\phi_{i}(\boldsymbol{x})$ onto the expansion basis $\tilde{\phi}_{i}(\boldsymbol{x})$ is represented by the derivative operator

$$
\tilde{\boldsymbol{M}}^{-1} \tilde{\boldsymbol{S}}_{l}
$$

where

$$
\tilde{\boldsymbol{M}}=\tilde{\boldsymbol{B}}^{T} \boldsymbol{W} \tilde{\boldsymbol{B}}, \quad \tilde{\boldsymbol{S}}_{l}=\tilde{\boldsymbol{B}}^{T} \boldsymbol{W} \boldsymbol{D}_{l} \boldsymbol{B}
$$


The spectral/hp element SVV operator is then imposed by applying a filter $\hat{\boldsymbol{Q}}$, which will be defined shortly, to the projected derivative of the basis implied by the matrix operation $\tilde{\boldsymbol{M}}^{-1} \tilde{\boldsymbol{S}}_{l}$. In directly analogy to equation (11) we can define the SVV operator (8) as

$$
\boldsymbol{S}_{V V}^{e}[i][j]=\epsilon\left(\sum_{l=1}^{\operatorname{Dim}} \tilde{\boldsymbol{S}}_{l}^{T} \hat{\boldsymbol{Q}} \tilde{\boldsymbol{M}}^{-1} \tilde{\boldsymbol{S}}_{l}\right)[i][j]=S_{V V}^{e}\left(\phi_{i}, \phi_{j}\right)
$$

Symmetry of discrete SVV System

We have yet to define any constraints on the modal expansion $\tilde{\phi}_{i}(\boldsymbol{x})$. For any expansion basis $\phi_{i}(\boldsymbol{x})$ the Galerkin projection leads to a weak Laplacian matrix $\boldsymbol{L}^{e}$ which is symmetric semi-positive definite. From a purely implementation point of view it is therefore attractive that the SVV system $\boldsymbol{S}_{V V}$ is also symmetric since the same matrix inversion techniques used on the Laplacian matrix can then be applied to the combined Laplacian and SVV system. From equation (14) we observe that the system will be symmetric providing that $\hat{\boldsymbol{Q}} \tilde{\boldsymbol{M}}{ }^{-1}$ is symmetric. As we shall see the definition of $\hat{\boldsymbol{Q}}$ is diagonal and so the symmetry of the SVV system can be enforced if $\tilde{\boldsymbol{M}}^{-1}$ is also diagonal.

In Karamanos and Karniadakis [8] the SVV filter was applied to the modal expansion coefficient of a semi-orthogonal expansion basis originally proposed by Dubiner [16], see also [3]. This basis was also used for the trial space of the spectral element expansion and so $\tilde{\phi}_{i}(\boldsymbol{x})=\phi_{i}(\boldsymbol{x})$. Although using the same basis for the SVV filter and elemental approximation is attractive from an implementation point of view, unfortunately $\boldsymbol{M}$ is not diagonal in this case and so the SVV operator is not symmetric.

To enforce the mass matrix $\tilde{\boldsymbol{M}}$ is diagonal necessarily implies that $\tilde{\phi}(\boldsymbol{x})$ is orthogonal (or at least discretely orthogonal). To be able to impose a diffusion on the high frequencies also implies that $\tilde{\phi}(\boldsymbol{x})$ should be modal/hierarchical even if the original basis $\phi(\boldsymbol{x})$ is nodal. For quadrilateral regions an appropriate orthogonal basis is the tensor product of the Legendre polynomials which has also been adopted in the spectral element SVV work of Xu \& Pasquetti [14]. An orthogonal expansion for the triangle, which is also the solution to a singular Sturm Liouville problem, also exists for the triangular region [3] and is defined in the next section.

\section{Definition of the filtering operator $\hat{\boldsymbol{Q}}$}

To complete our definition of $\boldsymbol{S}_{V V}$ in equation (14) we need to define the filtering operator $\hat{\boldsymbol{Q}}$. Before doing so we also require a definition of the ordering of the orthogonal expansion.

For a quadrilateral region in the standard space $\left\{-1 \leq \xi_{1}, \xi_{2} \leq 1\right\}$ an orthogonal expansion is the tensor product of Legendre polynomials $L_{p}(\xi)$ of the form,

$$
\tilde{\phi}_{i}(\boldsymbol{\xi})=\tilde{\phi}_{i(p, q)}(\boldsymbol{\xi})=L_{p}\left(\xi_{1}\right) L_{q}\left(\xi_{2}\right) \quad 0 \leq p, q \leq P .
$$


where $P$ is the polynomial order of the expansion in the $\xi_{1}$ and $\xi_{2}$ directions. In this definition we understand $i(p, q)$ to represent the consecutive ordering of the pair $(p, q)$ to the consecutive index $i$ which can be chosen as

$$
i(p, q)=p \times(P+1)+q .
$$

For a triangular region in the standard space $\left\{-1 \leq \xi_{1}, \xi_{2} ; \xi_{1}+\xi_{2} \leq 0\right\}$ an orthogonal expansion based upon a generalised tensor product can be defined as (see [3] for further details)

$$
\tilde{\phi}_{i(p, q)}(\boldsymbol{\xi})=P_{p}^{0,0}\left(\eta_{1}\right)\left(\frac{1-\eta_{2}}{2}\right)^{p} P_{q}^{2 p+1,0}\left(\eta_{2}\right) \quad 0 \leq p, q ; p+q \leq P
$$

where

$$
\eta_{1}=\frac{2\left(1+\xi_{1}\right)}{\left(1-\xi_{2}\right)}-1, \quad \eta_{2}=\xi_{2}
$$

and $P_{p}^{\alpha, \beta}(\xi)$ is the Jacobi polynomial and $P$ is the linear polynomial order of the expansion space. For this case we again understand $i(p, q)$ to represent the consecutive ordering from the pair $(p, q)$ to the matrix index $i$ which for this case be chosen as

$$
i(p, q)=\frac{1}{2} p(2 P+3-p)+q
$$

Having transformed our local approximation to this orthogonal expansion we once again apply a filter on all polynomial modes above a prescribed cutoff $P_{\text {cut }}$. We can therefore define a filter function as

$$
\hat{\boldsymbol{Q}}[i(p, q)][i(p, q)]= \begin{cases}0 & p+q \leq P_{\text {cut }} \\ e^{-\frac{(p+q-P)}{\left(p+q-P_{\text {cut }}\right)^{2}}} & p+q>P_{\text {cut }}\end{cases}
$$

The application of this type of filter to a tensor product expansion which spans a bilinear space typically means that more than $50 \%$ of all modes are modified by the filter. An alternative approach would be to filter modal contributions in a more tensor product type manner with a filter of the form

$$
\hat{\boldsymbol{Q}}[i(p, q)][i(p, q)]= \begin{cases}0 & p \text { and } q \leq P_{\text {cut }} \\ e^{-\frac{(p-P)(q-P)}{\left(p-P_{\text {cut }}\right)\left(q-P_{\text {cut }}\right)}} & \text { otherwise }\end{cases}
$$

The filter defined in equation (15) filters with respect to total polynomial order and therefore restricts the unaffected modes to the standard linear space (i.e. one level of a Pascals triangle). This filter can be applied to both triangular and quadrilateral expansions. However the filter (16) leaves a bilinear space unaffected and so can only be applied to a quadrilateral expansions which spans an appropriate bilinear space. All SVV simulations accomplished in this paper have adopted the filter given in equation (15). An alternative filter for quadrilateral elements 
which filters based upon the local elemental coordinate derivatives rather than the global coordinate derivatives has been proposed by $\mathrm{Xu} \&$ Pasquetti [14] which spans a similar space as the filter in equation (16). All these filters have the common feature that only modes higher than $P_{\text {cut }}$ are modified but the nature of the form of the high frequency decay has yet to be fully explored.

Positive Semi-Definiteness of $\boldsymbol{S}_{V V}^{e}$

To show that a matrix is positive semi-definite, we must demonstrate that

$$
\hat{\boldsymbol{u}}^{T} \tilde{\mathbf{S}}^{T} \hat{\mathbf{Q}} \tilde{\mathbf{M}}^{-1} \tilde{\mathbf{S}} \hat{\boldsymbol{u}} \geq 0
$$

for all non-zero real vectors $\hat{\boldsymbol{u}}$. We note that the equation (17) can be rewritten as:

$$
(\tilde{\mathbf{S}} \hat{\boldsymbol{u}})^{T} \hat{\boldsymbol{Q}} \tilde{\boldsymbol{M}}^{-1}(\tilde{\mathbf{S}} \hat{\boldsymbol{u}})
$$

Letting $\hat{\boldsymbol{v}}=\tilde{\mathbf{S}} \hat{\boldsymbol{u}}$, allows us to write this condition as

$$
\hat{\boldsymbol{v}}^{T} \hat{\mathbf{Q}} \tilde{\boldsymbol{M}}^{-1} \hat{\boldsymbol{v}} \geq 0 .
$$

Since $\tilde{\boldsymbol{M}}^{-1}$ is defined to be a diagonal positive definite matrix and $\hat{\mathbf{Q}}$ is a diagonal matrix in which all the diagonal entries are greater than or equal to zero (due to the choice of the filtering function) we know that $\hat{\boldsymbol{v}}^{T} \hat{\mathbf{Q}} \tilde{\boldsymbol{M}}^{-1} \hat{\boldsymbol{v}} \geq 0$ for all $\hat{\boldsymbol{v}}$.

\subsection{Demonstrative Numerical Experiments}

\subsubsection{One-Dimensional Inviscid Burgers Equation}

To demonstrate the influence of SVV, we first examine the standard Galerkin implementation of the inviscid Burgers equation with stabilisation given by:

$$
\frac{\partial u}{\partial t}+\frac{1}{2} \frac{\partial u^{2}}{\partial x}=S_{V V}(u)
$$

where we take the stabilisation term $S_{V V}(u)$ as the one-dimensional version of equation (7). In figure 1 we plot the solution of the inviscid Burgers equation at time $T=0.5$ with and without SVV. Five equally spaced element spanning $[-1,1]$ were used, each element containing $15^{\text {th }}$ order polynomials. In this example, a wave cutoff of $P_{\text {cut }}=7$ and amplitude of $\epsilon=1 / 16$ were applied.

Observe that the SVV has a favourable effect in that it greatly reduces the variation of the solution away from the discontinuity where the "wiggles" in the solution have been removed. 

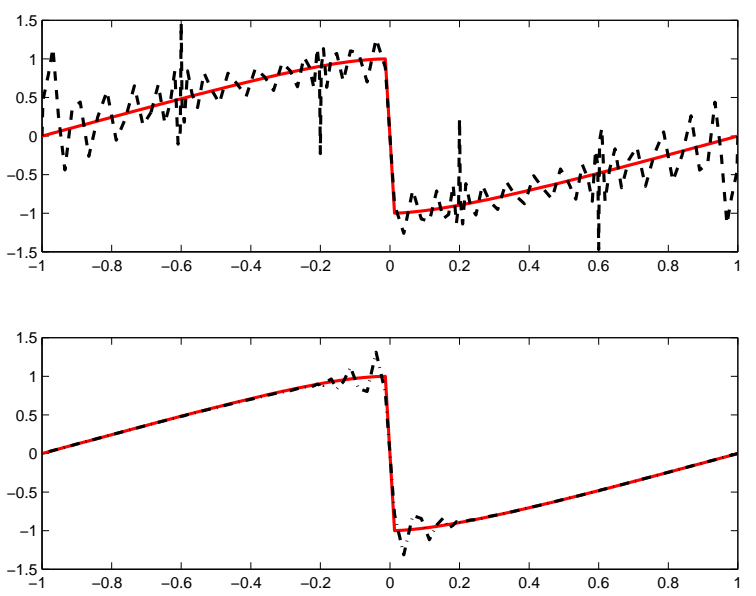

Fig. 1. Solution of inviscid Burgers equation at time $T=0.5$ using continuous Galerkin without SVV (top) and with SVV (bottom). Five equally spaced elements spanning $[-1,1]$ were used, each of which contained $15^{\text {th }}$ order polynomials.

This feature of SVV is consistent with the results shown for Fourier methods in [9] and for Galerkin spectral element methods in [8]. The SVV result away from the discontinuity looks very smooth as SVV dampens the propagating high frequency contribution generated at the sharp gradient.

\subsubsection{Diffusion of a Two-Dimensional Bubble}

To provide an example to reinforce our intuition about the influence of SVV when applied to multi-dimensional problems, we examine the following parabolic equation:

$$
\frac{\partial u}{\partial t}=\nu \nabla^{2} u+S_{V V}(u)
$$

on $[0,2] \times[0,2]$ with $\nu=10^{-5}$ and periodic boundary conditions.

To introduce high-frequency information into the initial condition a basic initial condition of $u(x, y, t=0)=\sin (\pi x) \sin (\pi y)$ was used applied with a discrete perturbation. To construct the initial condition the basis condition was projected onto the expansion space spanned by the spectral element discretisation and then re-evaluated at a discrete set of quadrature points. A perturbation was then introduced by explicitly setting the value at one point to a large magnitude as compared to its original value. In this example we set the function value at the point $(x, y) \approx(0.65,0.70)$ equal to 2.0 . This procedure is similar in concept to introducing a discrete delta function perturbation, and hence upon projection back to the spectral element function introduces energy into the high-frequency modes of the element containing the point of perturbation (and due to $C^{0}$ continuity may also cause propagation of the effect to neighbouring elements). For this example, a total of sixteen quadrilateral elements (four per direction) with $P=15^{\text {th }}$ order polynomials per element direction were used. Figure 2 shows the solution of equation (19) with and without SVV at time $T=0.1$. 
Figure 2 (left) shows the solution with no SVV; figure 2 (centre) shows the solution with SVV $\left(P_{\text {cut }}=7, \epsilon_{S V V}=0.1\right)$; and figure 2 (right) shows the solution with $\mathrm{SVV}\left(P_{\text {cut }}=3, \epsilon_{S V V}=\right.$ $0.1)$.
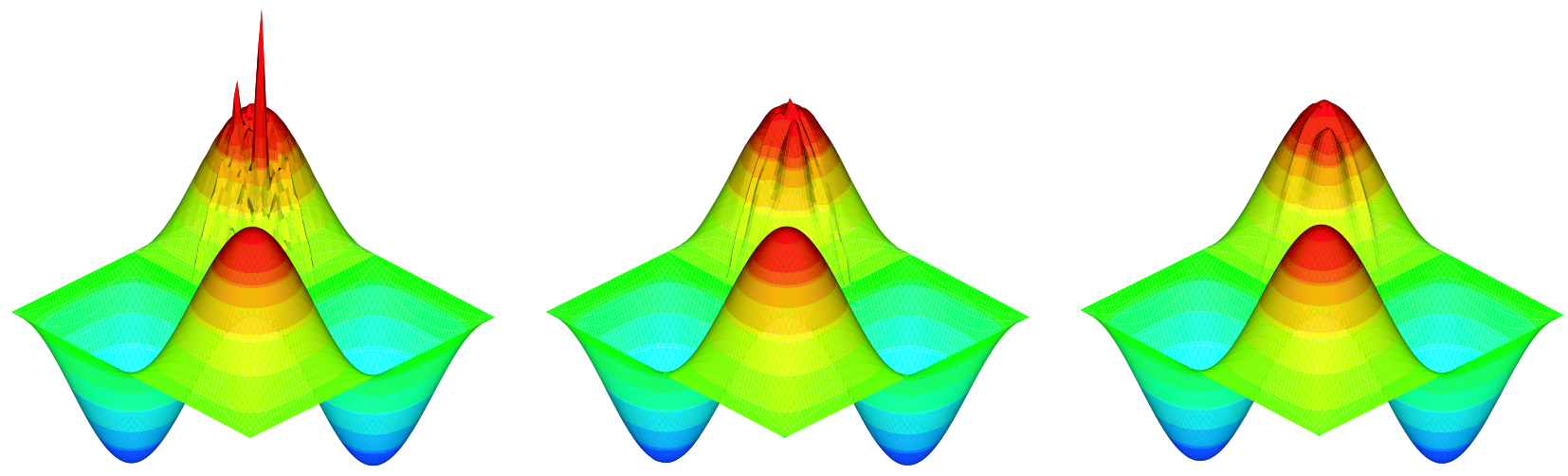

Fig. 2. Standard diffusion to time $T=0.1$ (left); standard diffusion with SVV $P_{\text {cut }}=7, \epsilon_{S V V}=0.1$ (centre); and standard diffusion with SVV $P_{\text {cut }}=3, \epsilon_{S V V}=0.1$ (right)

From this example we see that the SVV dissipation added to the high mode numbers with respect to the spectral element discretisation does indeed yield dissipation at the global high wavenumber scales of the solution (as exhibited in Figure 2 (centre and right)). Decreasing the SVV wavenumber cutoff $\left(P_{\text {cut }}\right)$ from eight to four produces further dissipation of the high wavenumber features within the solution.

\section{Incorporation of SVV into the Navier-Stokes Equations}

In this section we discuss how SVV can be incorporated into a velocity-correction splitting scheme to discretise the incompressible Navier-Stokes equations [17]. The incompressible Navier-Stokes equations can be written as:

$$
\begin{aligned}
\frac{\partial \mathbf{u}}{\partial t}+\mathbf{N}(\mathbf{u}) & =-\frac{1}{\rho} \nabla p+\nu \mathbf{L}(\mathbf{u}) \\
\mathbf{N}(\mathbf{u}) & =(\mathbf{u} \cdot \nabla) \mathbf{u} \\
\mathbf{L}(\mathbf{u}) & =\nabla^{2} \mathbf{u}
\end{aligned}
$$

The temporal discretisation adopted in this work is a projection scheme, based on backwards differencing in time. As originally described [17], this was characterised as an operatorsplitting scheme, but more recently [18] it has been shown that the method is one of a class of velocity-correction projection schemes.

The projection scheme requires the solution of a pressure Poisson equation to (approximately) maintain solenoidality of the velocity. Backwards time differencing is used to approximate a 
derivative at the new time level $(n+1)$ through

$$
\partial_{t}()^{(n+1)}=\frac{1}{\Delta t} \sum_{q=0}^{J} \alpha_{q}()^{(n-q+1)}+O(\Delta t)^{J+1},
$$

and in addition, a method is needed to explicitly extrapolate previous terms to the new time level, which is achieved through polynomial approximation

$$
()^{(n+1)}=\sum_{q=0}^{J-1} \beta_{q}()^{(n-q)}+O(\Delta t)^{J} .
$$

The discrete weights $\alpha_{q}, \beta_{q}$, for schemes of order up to $J=3$ appear in $[3,17]$.

The time-step for the velocity-correction scheme commences with the solution of a pressure Poisson equation, followed by a pressure-gradient update

$$
\nabla^{2} p^{(n+1)}=-\frac{\rho}{\Delta t} \nabla \cdot \boldsymbol{u}^{*}
$$

where

$$
\boldsymbol{u}^{*}=\sum_{q=1}^{J} \alpha_{q} \boldsymbol{u}^{(n-q)}-\Delta t \sum_{q=0}^{J-1} \beta_{q} \boldsymbol{N}\left(\boldsymbol{u}^{(n-q)}\right) .
$$

and augmented with a pressure boundary condition

$$
\partial_{n} p^{(n+1)}=-\rho \boldsymbol{n} \cdot \sum_{q=0}^{J-1} \beta_{q}\left(\boldsymbol{N}\left(\boldsymbol{u}^{(n-q)}\right)+\nu \boldsymbol{\nabla} \times \boldsymbol{\nabla} \times \boldsymbol{u}^{(n-q)}+\partial_{t} \boldsymbol{u}^{(n-q)}\right),
$$

where $\boldsymbol{n}$ is the domain unit outward normal. Equation (26) is an extrapolation of the Neumann pressure boundary condition on boundaries where no other condition is explicitly set. The enforcement of the solenoidality of $\nabla^{2} \boldsymbol{u}^{(n-q)}=\boldsymbol{\nabla} \boldsymbol{\nabla} \cdot \boldsymbol{u}^{(n-q)}-\boldsymbol{\nabla} \times \boldsymbol{\nabla} \times \boldsymbol{u}^{(n-q)}$ in forming equation (26) is essential to the time-accuracy of the scheme [17]. The step is completed by applying a viscous correction through the solution of a Helmholtz (elliptic) equation for $\boldsymbol{u}^{(n+1)}$

$$
\nabla^{2} \boldsymbol{u}^{(n+1)}-\frac{\alpha_{0}}{\nu \Delta t} \boldsymbol{u}^{(n+1)}=\boldsymbol{u}^{*}-\frac{\Delta t}{\rho} \nabla p^{(n+1)} .
$$

System (27) is actually, a set of scalar Helmholtz equations augmented with appropriate velocity boundary conditions at time $(n+1) \Delta t$. The use of higher derivatives in computing the rotational forms used in the pressure boundary conditions implies the use of a highorder spatial discretisation in the algorithm of [17], but other forms of the velocity-correction schemes do not carry this restriction, as pointed out in [18].

In our SVV implementation we start with the pressure Poisson equation given by equation (25) with boundary conditions given by equation (26). However the final Helmholtz step (27) is now augmented with a SVV operator as defined in (7) and so we solve

$$
\nabla^{2} \boldsymbol{u}^{(n+1)}+S_{V V}\left(\mathbf{u}^{n+1}\right)-\frac{\alpha_{0}}{\nu \Delta t} \boldsymbol{u}^{(n+1)}=\boldsymbol{u}^{*}-\frac{\Delta t}{\rho} \boldsymbol{\nabla} p^{(n+1)}
$$


with appropriate velocity boundary conditions at time $(n+1) \Delta t$. We note that in equation (28) the SVV term is scaled by the Reynolds number since the kinematic viscosity, $\nu$, which appears in the Helmholtz constant term scales both the diffusion and SVV terms. As discussed in section 2.2 we solve the pressure Poisson and Helmholtz equations using a standard Galerkin projection with a $C^{0}$ spectral/hp element spatial discretisation.

\section{Demonstrative Fluid Flow Examples}

In this section we provide three incompressible fluid flow examples, each of which demonstrate different aspects of the use of SVV. The incompressible fluid version of the spectral/hp element code $\mathcal{N} \boldsymbol{\varepsilon} \boldsymbol{\kappa} \mathcal{T} \boldsymbol{\alpha} \boldsymbol{r}[3,19]$ which is based on the continuous Galerkin method was employed for these simulations.

\subsection{Two-Dimensional Kovasznay Flow}

The first Navier-Stokes example we present is the solution of Kovasznay flow. The purpose of this example is to demonstrate that the addition of SVV as proposed does not destroy the expected convergence properties of the spectral/hp element method being employed. Kovasznay flow represented the laminar flow behind a two-dimensional grid [3], and is flow for which we have an exact solution to which to compare our numerical simulations. The steady-state solution is a function of the Reynolds number, $R e$, and is of the form

$$
\begin{aligned}
& u(x, y)=1-e^{\lambda x} \cos (2 \pi y) \\
& v(x, y)=\frac{1}{2 \pi} e^{\lambda x} \sin (2 \pi y)
\end{aligned}
$$

where $\lambda=R e^{2} / 2-\left(R e^{2} / 4+4 \pi^{2}\right)^{0.5}$. A rectangular domain given by $[-0.5,1] \times[-0.5,1.5]$ with Dirichlet conditions obtained from the above solution. Following [8], two domains were used, one consisting of eight quadrilateral elements and one consisting of two triangular elements as shown in figure 3. The computed steady-state streamline pattern for $R e=40$ is plotted in figure 3 (right).

To understand the influence of SVV on the convergence properties of spectral/hp methods, we undertook a parameter study for different polynomial orders and SVV parameters. The results are summarised in Table 1 where comparisons of the error at steady-state at $R e=40$ with and without SVV are shown. The two SVV parameters were computed as follows: $P_{\text {cut }}=(2 \sqrt{P+1})-1$ and $\epsilon_{S V V}=1 /(P+1)$.

Observe that SVV does modify the solution slightly, but in these reasonably resolved cases, it does not destroy the expected convergence. To further illustrate this fact, we plot in figure 

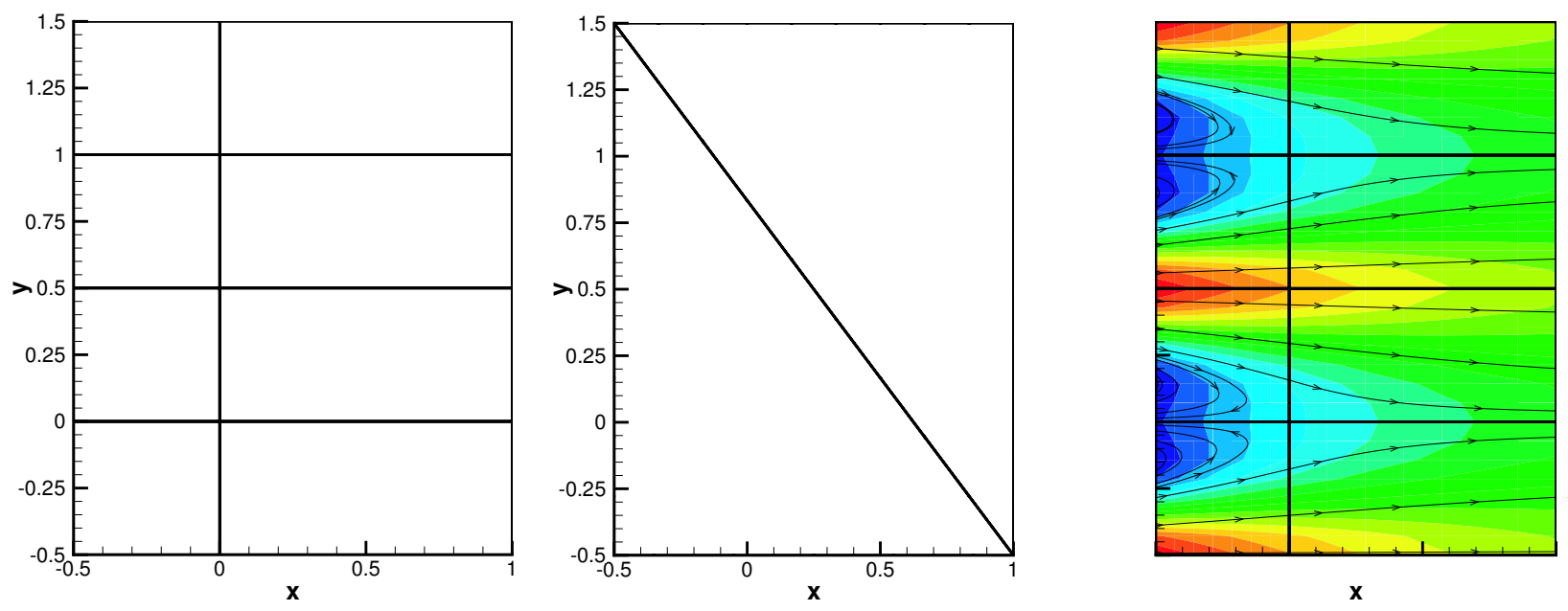

Fig. 3. Structured (left) and unstructured (centre) mesh used for the Kovasznay flow tests. Streamwise velocity contours with superimposed streamlines for $R e=40$ is presented on the right.

\begin{tabular}{||l|l|l|l|l|l|l||}
\hline Poly. Order $(P)$ & Elements & $\epsilon_{S V V}$ & $P_{\text {cut }}$ & $L_{\infty}$ Error & $L_{2}$ Error & $H_{1}$ Error \\
\hline 6 & 8 (Quads) & 0.0 & 0 & $2.41534 e-04$ & $7.94111 e-05$ & $2.25481 e-03$ \\
6 & 8 (Quads) & $1 / 7$ & 5 & $2.37631 e-04$ & $7.93659 e-05$ & $2.25958 e-03$ \\
\hline 8 & 8 (Quads) & 0.0 & 0 & $2.95956 e-06$ & $7.26612 e-07$ & $2.55962 e-05$ \\
8 & 8 (Quads) & $1 / 9$ & 6 & $2.39061 e-06$ & $7.73207 e-07$ & $2.83645 e-05$ \\
\hline 10 & 8 (Quads) & 0.0 & 0 & $2.49826 e-08$ & $4.50168 e-09$ & $1.81744 e-07$ \\
10 & 8 (Quads) & $1 / 11$ & 7 & $4.35637 e-08$ & $1.18875 e-08$ & $5.08203 e-07$ \\
\hline \hline 9 & 2 (Triangles) & 0.0 & 0 & 0.206259 & 0.0906154 & 0.816686 \\
9 & 2 (Triangles) & $1 / 10$ & 6 & 0.205844 & 0.0904501 & 0.814678 \\
\hline 15 & 2 (Triangles) & 0.0 & 0 & 0.00799273 & 0.00105305 & 0.0223986 \\
15 & 2 (Triangles) & $1 / 16$ & 8 & 0.00769555 & 0.00100951 & 0.0215664 \\
\hline 17 & 2 (Triangles) & 0.0 & 0 & 0.00174882 & 0.000179393 & 0.00453809 \\
17 & 2 (Triangles) & $1 / 17$ & 8 & 0.00168793 & 0.000171647 & 0.00436712 \\
\hline
\end{tabular}

Table 1

Effect of the SVV method on the Kovasznay solution using quadrilateral and triangular grids. All comparisons were done at steady-state for $R e=40$.

4 the $L_{2}$ error versus number of modes for the quadrilateral mesh. The SVV parameters are taken as previously mentioned. Observe that exponential convergence is still maintained even with the addition of SVV. Note that the slight staircase effect evident in the plot is due to the staircase nature of the integer parameter $P_{\text {cut }}$ (rounding is employed when exact integer values are not obtained, and hence a slight staircasing of the parameter results). 


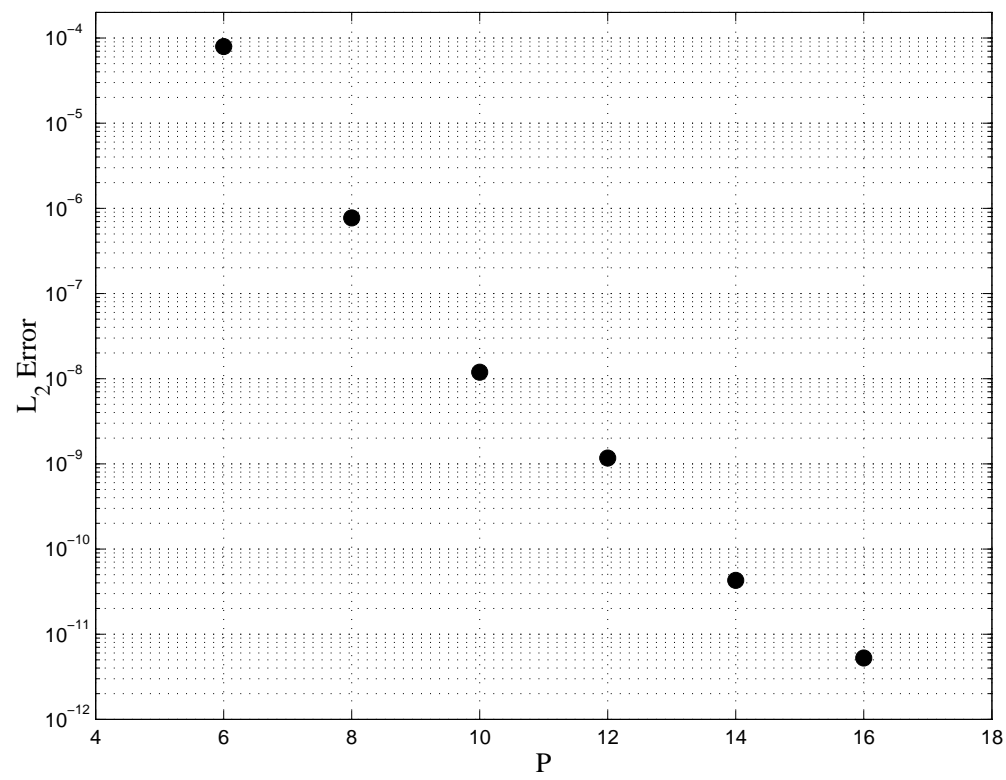

Fig. 4. Spectral (exponential) convergence is demonstrated for the exact Kovasznay Navier-Stokes solution using the SVV method.

\subsection{Two-Dimensional Double Shear Layer Flow}

To illustrate the stabilising effect of SVV, we consider the double shear layer problem examined in the context of under-resolution in [20,21]. The problem consists of solving the two-dimensional incompressible Navier-Stokes equations on a periodic box of length two (i.e. $[-1,1] \times[-1,1])$. The initial conditions are given as:

$$
\begin{aligned}
u(x, y)=\tanh (\epsilon(y+0.5)) & \{(x, y) \mid-1 \leq x \leq 1,-1 \leq y \leq 0\} \\
=\tanh (\epsilon(0.5-y)) & \{(x, y) \mid-1 \leq x \leq 1,0<y \leq 1\} \\
v(x, y)=\delta \cos (\pi x) & \{(x, y) \mid-1 \leq x \leq 1,-1 \leq y \leq 1\}
\end{aligned}
$$

where $\epsilon=40.0$ and $\delta=0.05$, and the kinematic viscosity is taken as $\nu=10^{-4}$. Given these initial conditions, the perturbed shear layers rolls up into two vortices with trailing arms. For the purposes of our numerical experiment, we consider an $8 \times 8$ evenly-spaced quadrilateral mesh, each element containing $15^{\text {th }}$ order polynomials. Using second-order time integration with a time step $\Delta t=0.0005$, we integrate the solution to final time $T=1.87$ upon which we can compare with the solutions in [21]. In Figure 5 (left) we present the solution the secondorder time integration with no SVV added. The solution remains stable and produces a result in agreement with [21].

If we reduce our time integration order, however, the solution becomes unstable (at a time $T<1.0$ ). This instability is to be expected since with the low viscosity of this problem we expect the imaginary eigenvalues of the advection operator to be dominant in the time 
stepping problem. Since the first order explicit time integration of the advection operator has a stability region which does not encompass the imaginary axis the solution becomes unstable. The purpose of this experiment is to see if SVV can be used to stabilise the solution without drastically destroying the anticipated result. Keeping all parameters the same but reducing the time integration order to first-order, we now introduce SVV with $P_{\text {cut }}=7$ and different values of $\epsilon_{S V V}$. In Figure 5 we show the SVV results with $\epsilon_{S V V}=5.0$ (centre) and $\epsilon_{S V V}=10.0$ (right).
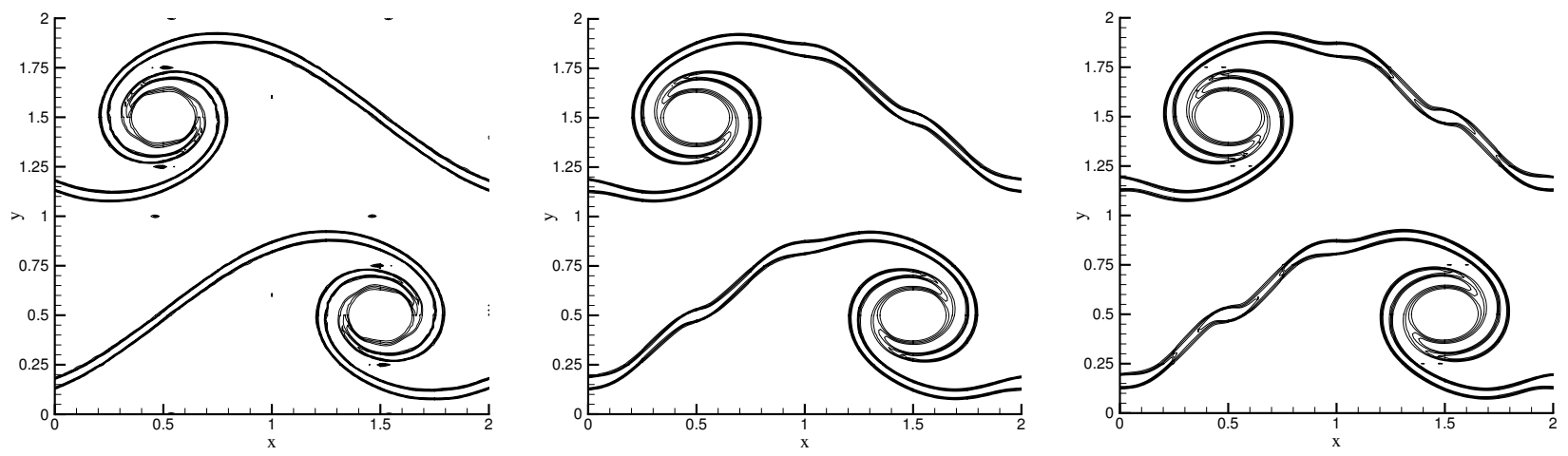

Fig. 5. Contours of vorticity at time $T=1.87$. (Left) second-order time integrator with no SVV; (centre) first-order time integrator with SVV enabled $\left(P_{\text {cut }}=7, \epsilon_{S V V}=5.0\right)$; (right) first-order time integrator with SVV enabled $\left.P_{\text {cut }}=7, \epsilon_{S V V}=10.0\right)$.

We note that the solution remains stable to time $T=1.87$ even with first-order integration which was previously unstable at $T<1.0$. Further, the SVV solutions look qualitatively similar to the original resolved solution. Note that the nature of the discrepancies between the SVV and non-SVV solutions are consistent with the under-resolution studies in [21]; SVV's removal of the high-frequency information produces a stable solution where the highfrequencies are under-resolved.

\subsection{Transition and Turbulence in a Three-Dimensional Triangular Duct}

In this third example, we demonstrate that SVV is not the solution to all problems which arise due to the lack of inconsistent quadrature. We consider the effect of inconsistent quadrature and associated aliasing errors by simulating transition to turbulence of incompressible flow in a duct with its cross-section being an equilateral triangle as previously studied in [2].

The laminar fully-developed solution is known analytically [3]. Random disturbances are introduced in the flow and then integrated in time until these disturbances start decaying or growing in time. All simulations were performed in the domain shown in figure 6 with the cross-section discretized using one triangular element and 16 Fourier modes the streamwise (homogeneous) direction. A duct length of three $D_{e}$ was used and the Reynolds number was defined as $R e=U D_{e} / \nu$ where $U$ is the average velocity and $D_{e}$ is the equivalent (hydraulic) diameter. For $R e \leq 500$ all disturbances decay, but for $R e=1250$ the flow goes through transition, and a turbulent state is sustained. Typical results are shown in figure 6 . 
We have performed four simulations at $R e=1250$ corresponding to different combinations of polynomial order $(P)$, quadrature order $(Q)$ and SVV parameters. In the first simulation, shown in figure 7 (a), we consider the case where $Q=P+2$, where $P=15$. The forces on the three walls of the duct are plotted as a function of time. From symmetry considerations, we expect that the statistical averages of the three forces are identical, but obviously the symmetry in the mean is not preserved here. In figure $7(\mathrm{~b})$ we plot the forces for the case with $Q=P+2$ with SVV employed $\left(P_{\text {cut }}=11, \epsilon_{S V V}=1 / 16\right)$, and in figure $7(\mathrm{c})$ we present the case with consistent-integration where $(Q=3(P+1) / 2)[2]$. From these three cases, we see that SVV as defined in this paper does not correct for the quadrature crimes committed in this example (in this case, the anisotropy of the quadrature point distribution upon which the collocated nonlinear terms are evaluated is the culprit); only consistent integration as presented in [2] rectifies the problem. A fourth case was also considered using the SVV parameters $P_{\text {cut }}=3, \epsilon_{S V V}=1 / 16$. This case demonstrated that employing more SVV does not yield a better solution.
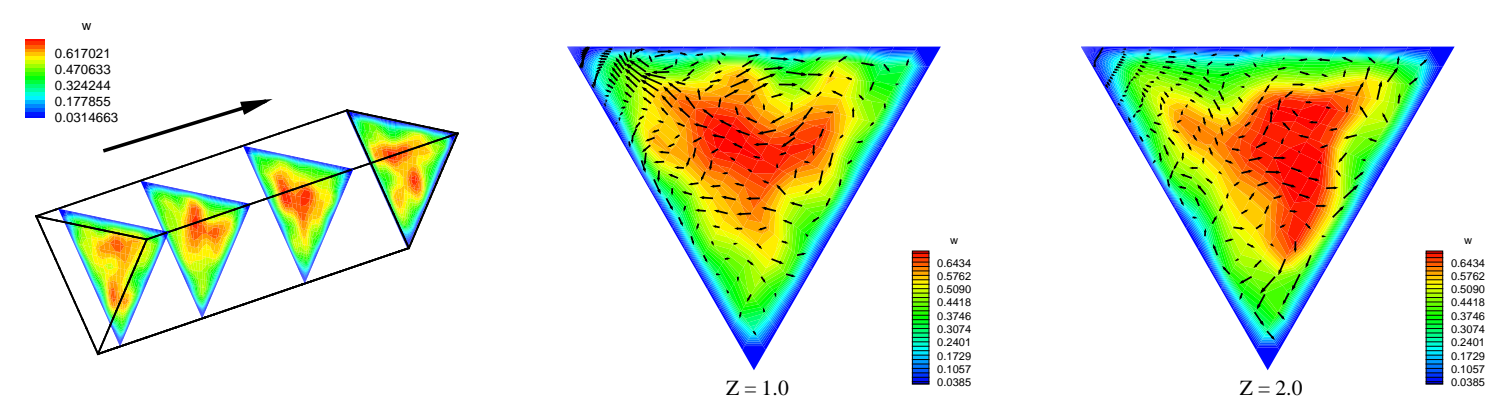

Fig. 6. Duct flow domain: The cross-section is an equilateral triangle and the streamwise length is three times the triangle edge. On the left we show a frame of the entire domain with flood contour cut-planes of the fluid velocity in the streamwise (w) direction. In the centre and on the right we present flood contour cut-planes of the fluid velocity in the streamwise (w) direction with arrows denoting the velocity in the crossflow $(\mathrm{u}, \mathrm{v})$ directions at $z=1$ and $z=2$ respectively.

In table 2, we present for all four cases the mean shear force calculated for each of the walls. Statistical averaging was started 40 convective units (over 13 duct lengths) after the initial perturbation period of 10 convective units; statistical averages were taken over 150 convective units (50 duct lengths). Only the consistent integration results provide the expected statistical results.

Table 2

\begin{tabular}{|c|c|c|c|}
\hline Quadrature Order & Wall 1 & Wall 2 & Wall 3 \\
\hline \hline$P+2($ no SVV $)$ & 0.0048 & 0.0054 & 0.0054 \\
\hline$P+2\left(\mathrm{SVV} ; P_{\text {cut }}=3, \epsilon_{S V V}=1 / 16\right)$ & 0.0048 & 0.0052 & 0.0053 \\
\hline$P+2\left(\mathrm{SVV} ; P_{\text {cut }}=11, \epsilon_{S V V}=1 / 16\right)$ & 0.0047 & 0.0054 & 0.0053 \\
\hline$\frac{3}{2}(P+1)($ no SVV $)$ & 0.0053 & 0.0053 & 0.0053 \\
\hline
\end{tabular}

Mean shear forces on each wall versus the quadrature order employed. 
(a)
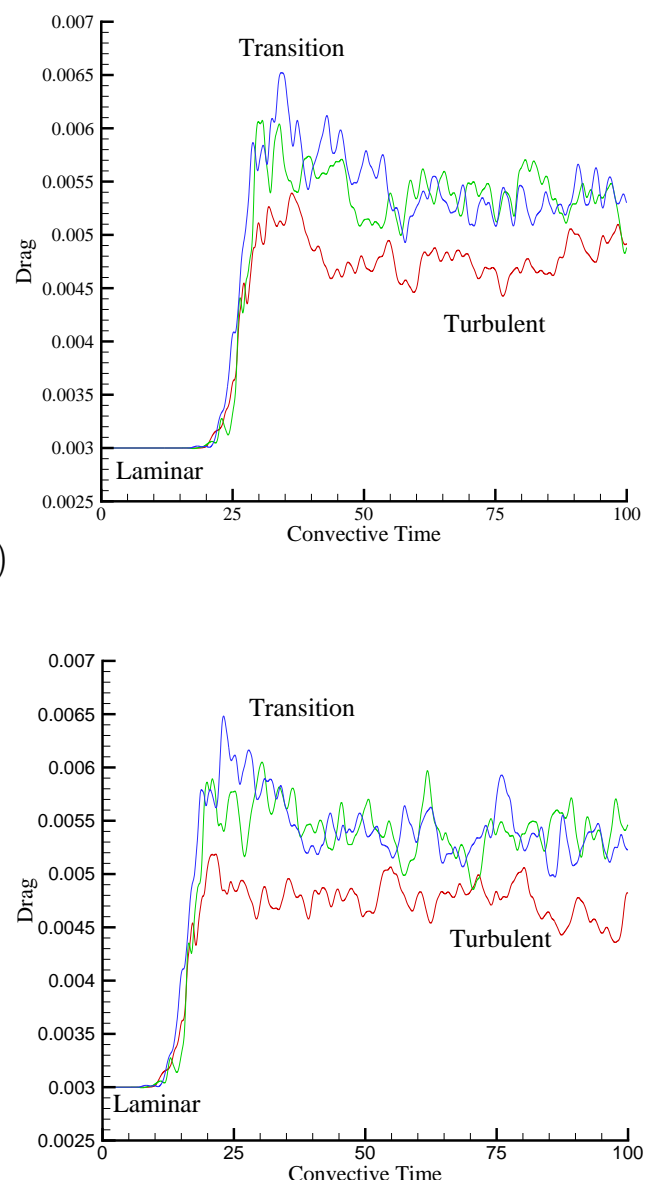

(b)

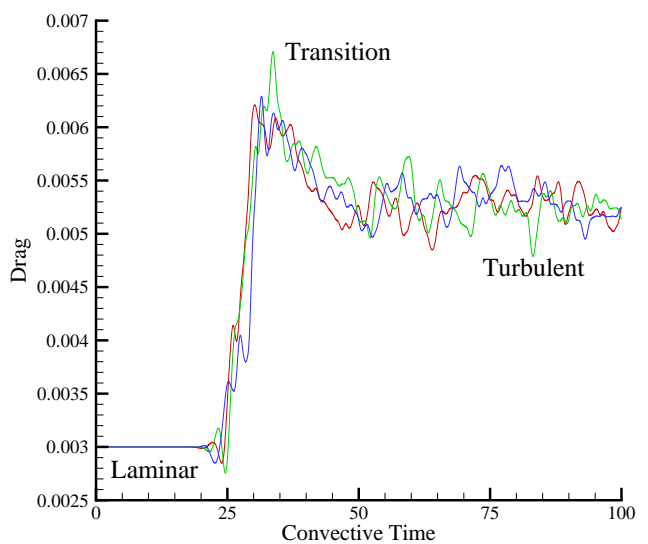

(c)

Fig. 7. Wall shear forces as a function of time for (a) $(Q=P+2)$, no SVV; (b) $(Q=P+2)$, SVV: $P_{\text {cut }}=11 ;$ and $(\mathrm{c})(Q=3(P+1) / 2)$, no SVV. 


\section{Summary}

In this paper we have presented a SVV formulation for quadrilateral and triangular multidimensional spectral/ $h p$ element methods based upon filtering with respect to an orthogonal basis. We have demonstrated that the formulation is symmetric and semi-positive definite. Through numerical flow examples and test cases we have shown that SVV does not degrade the exponential rate of convergence expected from spectral/ $h p$ element discretisation of smooth solutions. As also reported in previous papers $[8,14]$ we have observed that SVV can be used to stabilise solutions. We have also seen, however, that SVV is not necessarily a means of fixing all errors associated with problems such as consistent-integration. Nevertheless one aspect of the formulation which has not been fully investigated is the choice of different filter functions $\hat{\boldsymbol{Q}}$. As discussed in section 2.2.2 there are a variety of choices of $\hat{\boldsymbol{Q}}$ which can be considered, and we have observed that the filter matrix only needs to be positive and diagonal to ensure the symmetric semi-positive definite property of the discrete SVV operator. As discussed in [13] there is potential to design nonlinear (solution dependent) filters which could be applied in large eddy simulation. However we also note that a more judicious choice of $\hat{\boldsymbol{Q}}$ might lead to a better de-aliasing property for the triangular expansion applied in the last test case of turbulent flow in a channel. Nevertheless we can conclude that SVV provides another computational tool for stabilisation of spectral/ $h p$ element methods as applied to the incompressible Navier-Stokes equations.

\section{Acknowledgements:}

The authors thank both Dr. Hugh Blackburn of CSIRO, Australia, and Dr. George Karniadakis of Brown University, USA, for their remarks and helpful discussions. In addition, we thank Dr. Tim Warburton of Rice University, USA for providing detailed information concerning his published work on the shear layer example.

The first author gratefully acknowledges the computational support and resources provided by the Scientific Computing and Imaging Institute at the University of Utah.

The second author would like to acknowledge support from a Global Research Award from the Royal Academy of Engineering.

\section{References}

[1] J. von Neumann and R.D. Richtmyer. A method for the numerical calculation of hydrodynamical shocks. J. Appl. Phys., 21:380-385, 1950.

[2] Robert M. Kirby and George Em Karniadakis. De-aliasing on non-uniform grids: Algorithms and applications. J. Comp. Phys., 191:249-264, 2003. 
[3] G.E. Karniadakis and S.J. Sherwin. Spectral/hp Element Methods for CFD. Oxford University Press, 1999.

[4] D. Gottlieb and J. S. Hesthaven. Spectral methods for hyperbolic problems. J. Comput. Appl. Math., 128(1-2):83-131, 2001.

[5] J.S. Mullen and P.F. Fischer. Filtering techniques for complex geometry fluid flows. Comm. in Num. Meth. in Eng., 15:9-18, 1999.

[6] P.F. Fischer and J.S. Mullen. Filter-based stabilization of spectral element methods. In Comptes Rendus de l'Acadamie des sciences Paris, t. 332, - Srie I - Analyse numerique, pages 265-270, 2001.

[7] M.O. Deville, E.H. Mund, and P.F. Fischer. High Order Methods for Incompressible Fluid Flow. Cambridge University Press, 2002.

[8] G.-S. Karamanos and G.E. Karniadakis. A spectral vanishing viscosity method for large-eddy simulations. J. Comp. Phys., 162:22, 2000.

[9] E. Tadmor. Convergence of spectral methods for nonlinear conservation laws. SIAM J. Numer. Anal., 26(1):30, 1989.

[10] W.S. Don. Numerical study of pseudospectral methods in shock wave applications. Journal of Computational Physics, 110:103, 1994.

[11] M.G. Crandall and P.L. Lions. Viscosity solutions of Hamilton-Jacobi equations. Trans. Amer. Math. Soc., 61:629, 1983.

[12] Y. Maday, S.M. Ould Kaber, and E. Tadmor. Legendre pseudospectral viscosity method for nonlinear conservation laws. SIAM J. Numer. Anal., 30:321, 1993.

[13] Robert M. Kirby and George Em Karniadakis. Coarse resolution turbulence simulations with spectral vanishing viscosity - large-eddy simulations (SVV-LES). Journal of Fluids Engineering, 124(4):886-891, 2002.

[14] Chuanju Xu and Richard Pasquetti. Stabilized spectral element computations of high Reynolds number incompressible flows. J. Comp. Phys., 196:680-704, 2004.

[15] S. Sirisup and G.E. Karniadakis. A spectral viscosity method for correcting the long-term behavior of POD models. J. Comp. Phys., 194:92-116, 2004.

[16] M. Dubiner. Spectral methods on triangles and other domains. J. Sci. Comp., 6:345, 1991.

[17] G.E. Karniadakis, M. Israeli, and S.A. Orszag. High-order splitting methods for incompressible navier-stokes equations. Journal of Computational Physics, 97:414, 1991.

[18] J.L. Guermond and J. Shen. Velocity-correction projection methods for incompresible flows. SIAM J. Numer. Anal., 41:112-134, 2003.

[19] R. M. Kirby, T. C. Warburton, S. J. Sherwin, A. Beskok, and G. E. Karniadakis. The $\mathcal{N} \varepsilon \boldsymbol{\kappa} \mathcal{T} \boldsymbol{\alpha} \boldsymbol{r}$ code: Dynamic simulations without remeshing. In Proc. of the 2nd International Symposium on Computational Technologies for Fluid/Thermal/Chemical Systems with Industrial Applications, ASME PVP Division, Volume 397, 1999. 
[20] David L. Brown and Michael L. Minion. Performance of under-resolved two-dimensional incompressible flow simulations. Journal of Computational Physics, 122:165-183, 1995.

[21] T. Warburton, L.F. Pavarino, and J.S. Hesthaven. A pseudo-spectral scheme for the incompressible Navier-Stokes equations using unstructured nodal elements. J. Comp. Phys., 164:1-21, 2000. 